\title{
Mantle: An Open Source Platform for One Health Biosurveillance and Research
}

\author{
Andrew G. Huff ${ }^{* 1,2}$ and Toph Allen ${ }^{1}$ \\ ${ }^{1}$ EcoHealth Alliance, New York, NY, USA; ${ }^{2}$ Columbia University, New York, NY, USA
}

\section{Objective}

Mantle will be an open-source, cloud-compatible platform for storing, studying, and sharing data on infectious diseases across plants, animals, and humans. It will meet the needs of three groups of users: scientists, policymakers, and the general public. For scientists, Mantle will make datasets portable and connected. Scientists will be able to upload datasets to the Mantle website or collect data from the field using a mobile app. Users in Mantle will be able to easily make datasets entirely private, publicly accessible, or shared with specific users or groups.

\section{Introduction}

The One Health approach suggests that humans, animals, and the environment are closely tied together. Human interaction with wildlife and the environment contributes to increased risk for human, plant, and animal infectious disease outbreaks. Since human, animal, and ecosystem health are linked, interdisciplinary and holistic approaches are needed to prevent future infectious disease outbreaks. Despite the movement towards One Health, the software currently available to manage, analyze, and communicate the vast amount of One Health data is grossly inadequate. One Health data are continually growing in size and complexity, and new technologies must be developed to address the magnitude of the problem. Furthermore, the desire of single entities to control and leverage information for greater personal and organizational wealth and power directly opposes the goals of biosurveillance, One Health, and science. Open access and open source software are needed to address these complex One Health problems, and to improve data accessibility, interoperability, and information communication.

\section{Methods}

Mantle will handle tabular data, and other widely used spatial data formats. It will visualize and explore data in useful ways, and allow data to be downloaded as the originally uploaded file or in a customizable format for use in analytical software. Mantle will store metadata - information about a dataset and its contentsusing development standards for linked data (e.g., JSON-LD and WCSV, part of the overarching Resource Description Framework). Tapping into the emerging semantic web enables richer interactions with datasets, streamlining many common data tasks. Mantle will natively understand a number of data types common to One Health data, including spatial and temporal elements, taxonomic names, and case counts, and associates these with published ontologies. Mantle will also work seamlessly with any numeric, categorical, and textual data. Mantle will store metadata - information about a dataset and its contents-using development standards for linked data (e.g., JSON-LD and WCSV, part of the overarching Resource Description Framework). Tapping into the emerging semantic web enables richer interactions with datasets, streamlining many common data tasks. Mantle will natively understand a number of data types common to One Health data, including spatial and temporal elements, taxonomic names, and case counts, and associates these with published ontologies. Mantle will also work seamlessly with any numeric, categorical, and textual data.

\section{Conclusions}

Policymakers and decision makers will be able to view real-time visualizations of Mantle data feeds in dashboards. Researchers will be able to upload datasets representing the output of models built in other analytical software, which can be shared with policymakers, who can also view and interact with the output of custom-built modeling modules to view timely and meaningful summaries of public health data feeds. Potential use cases for the general public include browsing day-to-day textual and syndromic surveillance information, viewing the predictions of a one-time study, and monitoring the latest calculated epidemic curve in an outbreak or ongoing epidemic. Mantle will facilitate crosscutting collaborations between disciplines and institutions. Users will be able to create, manage, and join organizations and groups. Groups of users will access and collaborate on collections of datasets, grouped manually or by specified properties. For instance, users interested in Ranavirus can view and contribute to the Global Ranavirus Reporting System, a collaborative effort by scientists worldwide to aggregate observed cases of Ranavirus across species and locations (a Mantle prototype).

\section{Keywords}

Metadata; One Health Surveillance; Open Source; Data Sharing; One Health Research

*Andrew G. Huff

E-mail: andrewgeorgehuff@gmail.com 